\title{
Design and Implementation of Electric Steering Gear Inspection System for Unmanned Aerial Vehicles Based on Virtual Instruments
}

\author{
Xing ZHENG $^{1, a}$, He YANG $^{2}$ and Shao Jun LIANG ${ }^{1}$ \\ ${ }^{1}$ Wuhan Mechanical Technology College, Wuhan, 430075, China \\ ${ }^{2}$ Hubei University of Education, Wuhan, 430075, China
}

\begin{abstract}
A kind of UAV electric servo detection system based on Virtual Instrument is designed in this paper, including the hardware platform based on PC-DAQ virtual instrument architecture and the software platform based on LabVIEW function, structure and system implementation methods. The function, structure and system implementation method of software platform is also described. The gear limits checking, zero testing, time domain characteristics test results showed that the system achieves testing requirements well, and can complete detection of electric steering gear automatically, fast, easy and accurate.
\end{abstract}

Keywords. Virtual Instruments, Unmanned Aerial Vehicles, Electric Steering Gear, Inspection

\section{Introduction}

As an operational component of flight control system for an unmanned aerial vehicle, steering gear generates corresponding force and torque according to command of a flight control computer for manipulating steering panel or thrust guide mechanism, in order to control flight attitude and trajectory. Therefore, performances of the steering gear directly impacts performance and flight safety of the flight control system. In examining, debugging and maintaining the steering gear, it is necessary to comprehensively test the performances. To ensure that the unmanned aerial vehicle can smoothly and safely fulfill its flight task, performance of the steering gear shall be also tested before the vehicle takes off.

At present, electric steering gears of unmanned aerial vehicles are mostly manually inspected. In other words, in inspecting a steering gear, an inspector shall regulate position of potentiometer and switching state to generate various input signals such as analog and switch quantity signals necessary for exciting the steering gear. All input signals of the steering gear are indicated by an analog meter or manually observed. During testing, various performance parameters, including startup current, operating current, startup voltage and times of magnification, shall be manually read and recorded by the inspector. Such manual inspection has some problems like difficulty for testing, low efficiency and poor precision. To solve difficulties for inspecting an electric steering gear, it is necessary to develop an electric steering gear inspection system for the unmanned aerial vehicle.

\section{System Development Plan}

\footnotetext{
${ }^{a}$ Xing ZHENG: zhengxcn@foxmail.com
}

Based on full demonstration, a detailed development plan is made. Built up with virtual instruments and technologies, the system is made up two major parts, including hardware and software platforms. Virtual instruments belong to a system of computer instruments designed and designed by users with a virtual panel on a computer-based hardware platform and test functions realized by testing software. In essence, they simulate interfaces of traditional instruments by computer display, to output measurement results in multiple forms. Data of signals are calculated, analyzed and processed with computer software. Meanwhile, signals are acquired, measured and conditioned by I/O equipment, in order to make availability of all testing functions.

Being fundamental for virtual instruments, hardware platform is mainly responsible for signal acquisition, amplification, analog-to-digital conversion and display and so on. With a PC-DAQ virtual software architecture, system hardware is composed of multi-functional data acquisition card, signal conditioning board and power supply.

Being critical for virtual instruments, software platform is primarily used for calculating, analyzing and processing data of tested signals. System software is developed by LabVIEW graphic programming language. Data are managed and conditioned by Microsoft SQL Server, including tested items, parameter processing, test case generation, system management, databases and other functional modules.

\section{Development Plan for Hardware Platform}


In consideration of reliability, openness, scalability, portability, standardization and requirements for speed and functions, system hardware is designed with PCDAQ virtual instrument architecture pursuant to practical testing requirements, made up of portable computer, data acquisition card, signal conditioning board and power supply. The components of system hardware are shown in Fig 1 as follows.

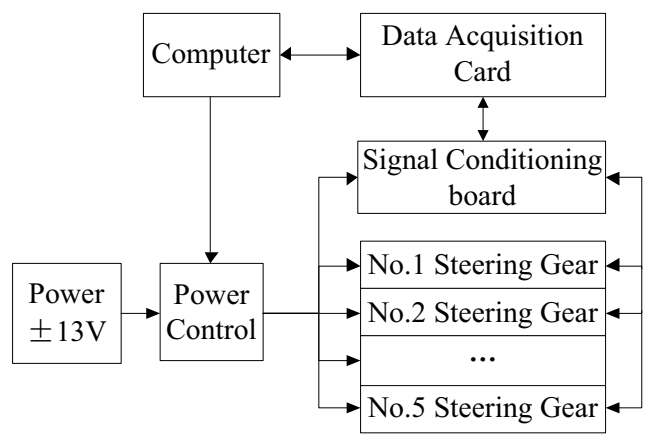

Figure 1. Components of Hardware Platform

\subsection{Portable computer}

In view that the system shall be portable and integrated, a portable computer is used as computer platform of the system. Required to be designed with current common basic configuration, this system has a socket for PCMCIA bus.

\subsection{Data acquisition card}

NI's DAQCard-6024E data acquisition card is used. As one of E-series plug-and-play multi-functional data acquisition cards based on PCMCIA bus, it has 16 channels of analog inputs with a sampling frequency of $200 \mathrm{KS} / \mathrm{s}, 2$ channels of analog outputs with a sampling frequency of $10 \mathrm{KS} / \mathrm{s}, 2$ 24-bit timer/counter and 8 digital $\mathrm{I} / \mathrm{O}$ ports that support TTL.

\subsection{Signal conditioning board}

As an intermediary module that connects computer, power supply and steering gear, it mainly includes sampling/follower module, current sampling module and power control module for sampling output current signals and controlling working power of steering gear.

\subsection{Power supply}

Power is supplied by constant-voltage supply and dualredundant power supply of nickel-cadmium battery pack, on the other hand, to provide multi-channel DC power supply for the signal conditioning board, on the other hand, to make high-power working power available to the steering gear.

\section{Development Plan for Software Platform}

Developed by LabVIEW graphic programming language, software platform is made up of several functional modules, including tested items, parameter processing, test case generation, system management and databases. From Fig 2, it may be discovered that database is essential for the software of that system, where data are organized and managed through databases. To meet the requirements for different tested items, software modules "Tested Items and Parameter Processing" are set during software design. Tested items and relevant parameters are set by a tester according to different functional requirements. Set content is stored in the database of the tested system, in order that test cases may be directly transferred from the database for subsequent tests. In this way, it is unnecessary to set tested items and parameters every time. It is convenient to uniformly manage data in a database, and acquired data may be stored in hard disk drive in the form of data files.

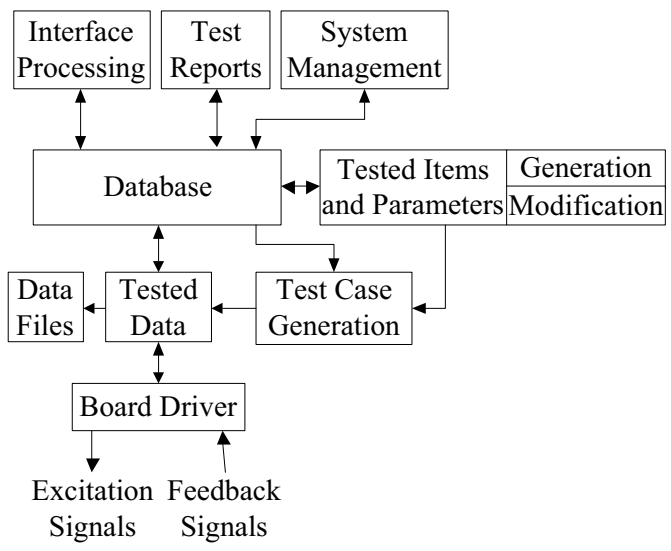

Figure 2. Components of Software Platform

Database is an indispensable part of data processing system in a computer. In organizing numerous data, they may be efficiently managed by database technologies. Waveform files or arrays are available from LabVIEW for storing acquired data. Frequency of excitation and feedback signals isn't quite high in the tested steering gear, so data are stored in the form of a database for convenient management. The database is operated in LabVIEW by ADO database interface technologies through ActiveX.

\section{Specific Implementation}

The system testing process is shown in Fig 3 as follows. Fig 3 shows the main program flowchart of tested system software, from which it may be discovered that the whole tested system is designed into multiple modules in terms of software, including modules of initialization and test case generation, in order to be in line with basic design principles of software engineering. A test may be performed once the testing program is started up, and test 
results may be replayed. To test software, created test cases may be used or directly manually tested, while basic or combinational test cases may be stored in a database. Test results may be stored in databases and data files. In the mean time, they are displayed.

In performing a test, test cases are needed at first. Type of steering gear and distribution of $\mathrm{I} / \mathrm{O}$ ports are concerned about identification of tested data. To choose type of a controller, provided that type of the steering gear is given and $\mathrm{I} / \mathrm{O}$ is defined, corresponding control panel and program interface will be available from the program. Then, users can use existed test cases in the database and make modifications based on that, or input the test cases by hands. Once the test cases are judged to be legitimate, they will be added to the database and initialized (including database and board of the test).

The test may be performed once the test cases are determined. It is divided into manual and automatic tests. During testing, sampling waveforms may be checked on a real-time basis (occasionally time-delayed). It is possible to change excitation signals during testing and ensure the test is performed on a real-time basis. A control panel will be available from the program to change inputs of a controller by hands on a real-time basis. For an automatic comprehensive test, a test case editor will be provided. Once the test is started, data will be automatically tested and then collected pursuant to cases.

In this paper, data of $\mathrm{I} / \mathrm{O}$ ports are divided into analog quantity, switching value and digital value (serial codes or pulse sequence). For input signals of the controller, excitation signals are synchronously provided by analog quantities of each channel, and switching values of all channels are in sync with the level translation. Sampling signals are sampled at certain frequency according to characteristics of separate channel signals, and generally synchronously sampled. To save space, different signals are organized in distinct ways. For instance, for analog signals, it is only necessary to record values of all sampling points after recording starts or ends, because the sampling frequency is constant in each channel. As regards switching value, only initial level and time of level changes are recorded.

To display data, oscilloscope, data sheets and analog display models of panels will be provided. For switching value and analog quantity, waveforms may be displayed in an oscilloscope, while digital value is marked at the response time.

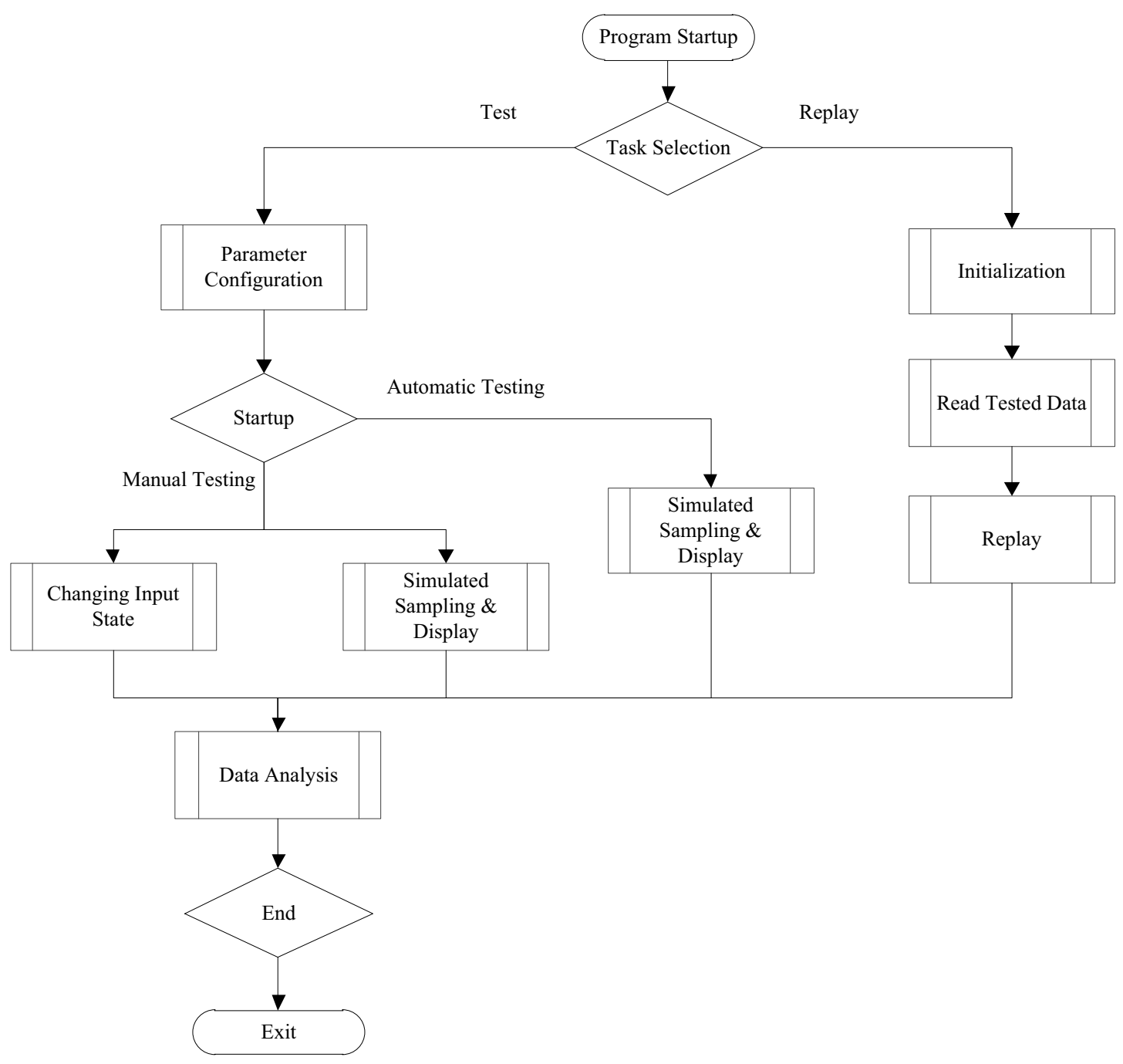

Figure 3. Flowchart of Tested Main Program 


\section{Results}

Generally, electric steering gear of an unmanned aerial vehicle stably controls flight by following. According to requirements for static and dynamic performances of the steering gear, tested items are confirmed to mainly include limit check, zero-bit test, zero-modulation test, following, time-domain and frequency-domain characteristics. The electric steering gear inspection system for unmanned aerial vehicle designed by above methods is used for inspecting major tested items. Fig 4, 5 and 6 respectively show polarity test results, zero-bit test results and analysis of time-domain characteristics. All results suggest that inspection requirements are reasonably met and inspection purposes are fulfilled.

\section{References}

1. Li Ying, Yu Jianguo, Shao Kaifeng, Automatic Test System of Electric Helm Gear Based on Single Chip
Microcomputer \& PC, Computer Measurement \& Control, Vol.16, 955(2008)

2. $\mathrm{Su} \mathrm{Na}$, Development and application of virtual instrument LabVIEW, Computer CD Software and Applications, Vol.23, 131(2013)

3. Yan Gang, Zhou Jun, Design and Application of Steering Gear Angle Detection System, Electronics World, Vol.16, 141(2013)

4. Lu Xiaoli, Research on Automatic Test Method of Linear Actuator, Journal of Mudanjiang College of Education, Vol.4, (2007)

5. Jiang Yang, Cong Shuquan, Zhu Wenlai, Design and Implementation of Intelligence Test System for Electric Actuator Based on LabVIEW, Journal of Astronautic Metrology and Measurement, Vol.31, 65(2011)

6. Zeng Man, He Weiguo, Systematic Implementation of Steering Engine Comprehensive Performance Test Platform, Computer Measurement \& Control, Vol.18, 1518(2010)

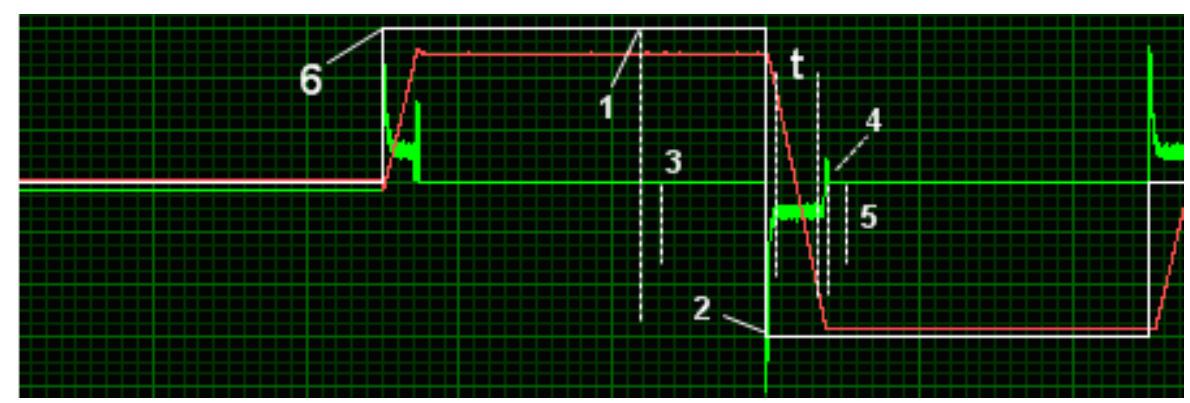

Figure 4. Curves of Polarity Tests (White: Excitation; Red: Feedback; Green: Current)

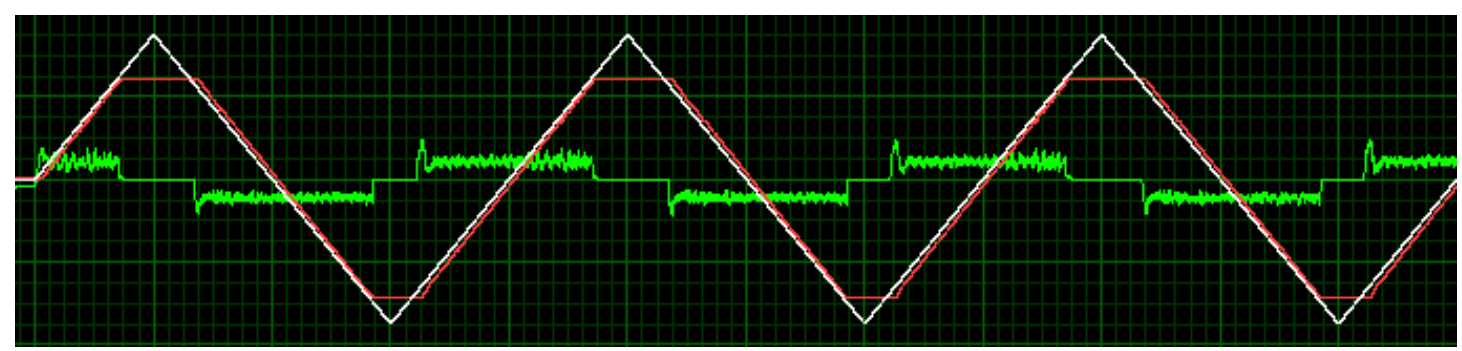

Figure 5. Curves of Zero-bit Tests (White: Excitation; Red: Feedback; Green: Current)

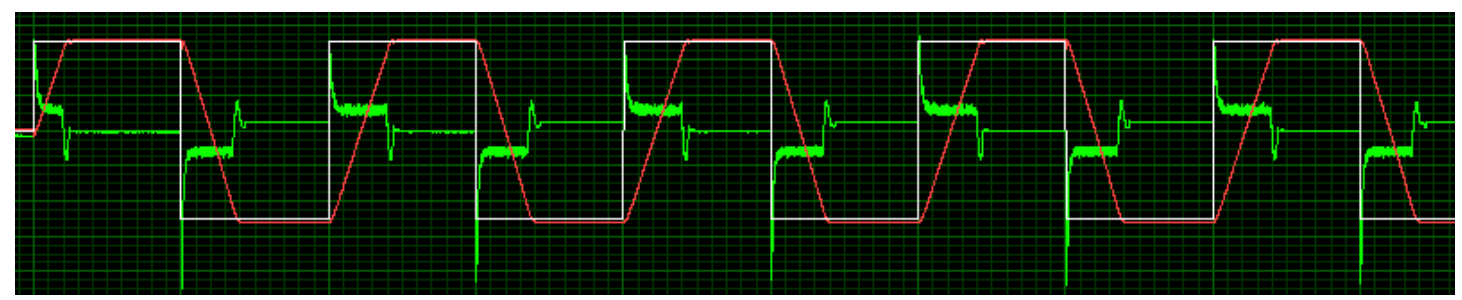

Figure 6. Waveforms for Analyzing Time-domain Characteristics (White: Excitation; Red: Feedback; Green: Current) 